УДК 622.691 .24

\title{
ИССЛЕДОВАНИЕ ЭФФЕКТИВНОСТИ ИНГИБИТОРОВ ГИДРАТООБРАЗОВАНИЯ В ПРОЦЕССЕ ДОБЫЧИ И ТРАНСПОРТА УГЛЕВОДОРОДОВ В МОРСКИХ УСЛОВИЯХ
}

\author{
Китаев Сергей Владимирович1, \\ svkitaev@mail.ru
}

\author{
Колотилов Юрий Васильевич², \\ kolotilov_yury@mail.ru
}

Плотников Антон Юрьевич2, plotnikovyurii@rambler.ru

\author{
Ковалев Адель Ахмедович², \\ truenerd123@gmail.com
}

\author{
Шейхгасанов Шамсутдин Кадиевич 1 , \\ sheih0113@mail.ru \\ 1 Уфимский государственный нефтяной технический университет, \\ Россия, 450062, г. Уфра, ул. Космонавтов, 1. \\ 2 Астраханский государственный технический университет, \\ Россия, 414056, г. Астрахань, ул. Татищева, 16.
}

\begin{abstract}
Актуальность исследования обусловлена необходимостью разработки эфффективных методов предотвращения гидратообразования в подводных газопроводах. Быстрому образованию газовых гидратов способствует высокое давление и низкая температура в глубоководных подводных сооружениях, что является одной из наиболее сложных проблем обеспечения работоспособности трубопровода. Основным направлением обеспечения безаварийной работы трубопроводов является поддержка процессов использования ингибиторов гидратообразования при эксплуатации объектов транспорта углеводородного сырья с учетом выделения их достоинств и недостатков.

Цель: разработка метода оценки эффективности ингибиторов гидратообразования в процессе добычи и транспорта уәлеводородов в морских условиях.

Объекты: эксплуатационные показатели реализации методов использования ингибиторов гидратообразования с оценкой риска, связанного с образованием гидратов, которая помогает предотвратить закупорку гидратов и обеспечить целостность оборудования.

Методы. Для повышения достоверности интерпретации методов применения ингибиторов гидратообразования предлагается использовать программное обеспечение OLGA, которое представляет собой инструмент моделирования анализа перекачки нефрти, природного газа и пластовой воды при многофразной транспортировке. Этот инструмент моделирования позволяет визуализировать ключевые аспекты динамических событий, отражающих реальность транспортировки углеводородов. Пакет программ OLGA позволил разрабатывать месторождения нефрти и газа в более глубоких водах и дальше от берега, чем это было бы возможно без этой технологии.

Результаты. Использование программного обеспечения OLGA позволило рассмотреть существующие методы предотвращения гидратообразования и сделать вывод о том, что технология холодного потока, а также добавление 10\%-го раствора метанола являются наиболее выгодным решением для Арктики по сравнению с другими технологиями и другими концентрациями соответственно.
\end{abstract}

\section{Ключевые слова:}

Морской трубопровод, ингибитор, гидратообразование, программное обеспечение, концентрация, давление, температура.

\section{Введение}

Обеспечение потока - один из самых важных аспектов в нефтяной и газовой промышленности и служит целью того, что сырая нефть и природный газ транспортируются экономно с месторождения на установки подготовки. В основном углеводороды транспортируются от места добычи до береговых или морских мест подготовки посредством трубопроводов и часто бывает так, что течение в трубопроводах многофазное. В дополнение к этому гидраты (подобная льду структура, где газовая молекула заключена в ячейку молекулы воды) часто формируются в трубопроводах и закрывают проходное сечение, замедляя добычу, и приводят к дорогостоящим операциям по ремонту.
При благоприятных условиях газовые гидраты могут образовываться в добывающих скважинах, в сборных промысловых трубопроводах и коллекторах, в магистральных газотранспортных системах, в трубопроводах установок предварительной подготовки и переработки газа [1-3].

Закупорка трубопровода - одна из основных проблем в нефтегазовой промышленности, так как это приводит к дорогостоящему ремонту. Хотя термодинамика гидратов была изучена в течение многих десятилетий, исследование переходного характера образования гидратов в трубопроводах довольно новое и его начали проводить относительно недавно. Во избежание формирования гидратов и для обеспечения потока выбираются специальные методы. 
Образование газогидратов в подводных магистральных трубопроводах является основной проблемой обеспечения потока в глубоководных трубопроводах и указывается в качестве основной технической проблемы в развитии морской энергетики. Обычно образование газогидратов в нефте- и газопроводах предотвращается введением большого количества ингибиторов, таких как метанол или гликоли [4-6]. Как альтернатива, образование газовых гидратов можно контролировать, используя небольшие количества полимерных соединений (кинетические ингибиторы), которые вместо ингибирования образования работают термодинамически, замедляя скорость формирования газовых гидратов.

Однако использование кинетических ингибиторов гидратов требует детальных знаний о зародышеобразовании и росте газовых гидратов и о том, как на эти процессы влияют давление и температура по отношению к фазовым равновесиям.

Большинство существующих методов обеспечения потока газа направлены:

1) на поддержание условий эксплуатации трубопровода за пределами зоны стабильности гидрата (например, изоляцией или дистанционным нагревом) ¡7-9];

2) предотвращение образования гидратов путем введения термодинамических ингибиторов (например, метанола или этиленгликоля) [10, 11];

3) достаточную задержку зарождения или роста гидрата путем введения кинетических ингибиторов гидратообразования ¡12-14];

4) снижение содержания воды [15-17].

Однако все эти способы становятся менее практичными из-за их высокой стоимости или ограничения, особенно в глубоких водах и при больших обводнениях. Поэтому отрасль переходит к управлению образованием гидратов. Таким методом является Cold Flow $[18,19]$, то есть холодный поток, который имеет несколько общих идей, таких как отсутствие нагревания или изоляции, гидраты не предотвращаются, но могут образовываться.

Гидраты, как правило, образуются в трубопроводах с природным газом при наличии воды и благоприятных условиях температуры и давления, как правило, при низких температурах и соответствующих высоких давлениях. Агломерация гидратов [20-22] может привести к блокировке трубопроводов и оборудования [16]. Следовательно, требуется тщательная оценка риска, связанного с образованием гидратов, которая помогает предотвратить закупорку гидратов и обеспечить целостность оборудования.

\section{Проблемы обеспечения потока во время добычи} или транспорта углеводородов

Обеспечение потока - это очень важная часть, которая гарантирует достижение требуемых показателей жизненного цикла системы от призабойной зоны к разгрузочным резервуарам путем прогнозирования, предотвращения и решения проблем, возникающих в результате поведения транспортируемых веществ (газов, жидкостей в разделенных либо в многофазных условиях).
Акцент на обеспечение потока имеет некоторые физические границы. Несомненно, всякий раз, когда поток из пласта ограничен, нормальное обеспечение потока начинает становиться проблемой. Это может происходить в трубах любого типа (например, в скважинных трубопроводах, отводных трубопроводах или транспортных трубопроводах). Общим для всех этих мест является то, что даже крошечные превращения, производимые жидкостями, могут оказывать сильное влияние на работоспособность и отличаться от желаемого потока.

Углеводороды присутствуют либо в парообразной, либо в жидкой форме (или в обеих), но также будут присутствовать пластовая вода, вода для закачки, песок, продукты коррозии, строительный мусор, асфальтены, частицы и другие компоненты [23].

На мелководье или на суше получить доступ к стволу скважины и трубопроводам проще. По мере того как операторы перемещаются в более отдаленные районы, на более глубокие морские глубины, они сталкиваются с трудностями в поддержании потока добываемых жидкостей и газа. Глубоководные стволы скважин и трубопроводы более сложны для доступа и представляют большую сложность в избежание или предотвращении закупорки.

Наиболее важной технической проблемой, с которой сейчас сталкивается нефтяная промышленность, является обеспечение потока. В рамках обеспечения потока проблемы с гидратами газа представляют собой наиболее серьезную угрозу для транспортировки нефти и газа по трубопроводам. Скопления гидратов, которые закупоривают трубопроводы, могут нарушить нормальные производственные графики и привести к экономическим потерям или даже создать риск безопасности, если не будут должным образом предупреждены или предотвращены. Проблема гидратов в трубопроводах обходится в миллиарды долларов для газопроводов.

Чтобы определить условия потока для образования гидрата, нам нужно рассчитать давление и температуру вдоль трубопровода, изучая динамику жидкости и тепломассопереноса. Оценка падения давления в трубопроводе играет важную роль в проектировании трубопровода и гарантирует бесперебойную транспортировку продукта.

Формирование гидратных пробок в трубопроводе занимает всего несколько часов [24] и приводит к нарушению технологического процесса. Очень важно распознать образование гидратных пробок, прежде чем ситуация ухудшится. Как правило, программные данные показывают, что в трубопроводе есть колебания давления до образования гидратной пробки. Пики на кривой давления и времени предупреждают о сильном отложении гидрата на стенке трубопровода.

Теоретически, если вся вода будет удалена из потока природного газа, условия для образования гидрата не будут выполняться. Оффшорное обезвоживание может быть неосуществимым для операций из-за физических ограничений на производственный объект на морских объектах. Также может не быть необходимости иметь морскую установку для обезвожи- 
вания, если стояки внизу морского дна не имеют крутых склонов, но температура и давление в трубопроводе так же важны, как и наклон райзера. При этом обезвоживающие сооружения не являются наиболее экономически эффективным способом предотвращения образования гидратов в подводных транспортных трубопроводах

С точки зрения эксплуатации, если поддерживать параметры потока за пределами кривой гидратного равновесия, это также предотвратит гидратообразование. Для достижения этих условий могут быть применены термодинамические, кинетические ингибиторы и тепловые методы. Управление кривой гидратообразования может быть достигнуто путем введения ингибиторов в трубопровод. Некоторые термические методы используются для того, чтобы обеспечить безвредное для окружающей среды управление кривой гидратообразования без подачи химикатов в трубопровод.

Традиционные способы обеспечения потока газа это удаление элементов, которые способствуют образованию гидрата. Гидратообразования можно избежать путем гидратации, которое уменьшает количество воды, доступной для образования гидрата, или путем поддержания рабочего давления и температуры вне области образования гидрата. Последний метод основан на смещении гидратной фазовой границы за пределы рабочих условий (давление и температура) во время нормальной добычи или транспорта. Этого можно достичь, сдвинув гидратную фазовую границу системы влево, добавив химические соединения, такие как термодинамические ингибиторы, сместив рабочую линию вправо, нагревая или изолируя трубопровод. Также возможно сместить рабочую линию за пределы зоны стабильности гидрата, контролируя рабочее давление (хотя этот параметр обычно используется для диссоциации гидратной пробки путем сброса давления).

Существуют различные методы обеспечения потока для решения проблемы образования гидратов в трубопроводах. Некоторые методы связаны с предотвращением образования гидратов, в то время как новая тенденция заключается в том, чтобы допускать образование гидратов, но предотвращать образование гидратной пробки.

\section{Добавление ингибиторов гидратов в систему}

Современные решения для предотвращения образования гидратов в основном осуществляются с помощью термодинамических средств, то есть поддержания температуры и давления на безопасном уровне

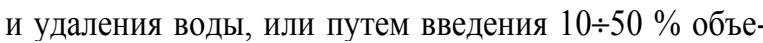
ма термодинамических ингибиторов, таких как моноэтиленгликоль, в жидкость. В дополнение к термодинамическим ингибиторам в последнее десятилетие были разработаны новые методы, такие как кинетические ингибиторы и антиагломеранты, чтобы предотвратить рост кристаллов гидратов и их агломерацию соответственно. В этом методе полимеры и поверхностно-активные вещества добавляются в по- ток в трубопроводах, образуя эмульсии (вода-в-масле) или микроэмульсии.

Для минимизации риска образования гидратов обычной практикой является использование избыточного количества ингибитора. Чрезмерная дозировка ингибитора приводит к негативным воздействиям на окружающую среду и нежелательным дополнительным расходам. По этой причине необходимость минимизировать количество ингибиторов является еще одной мотивацией для продолжения исследований по определению механизма гидратообразования.

Наиболее распространенным методом для предупреждения гидратов, применяемым в промышленности в настоящее время, является применение ингибиторов.

Разработка экономически эффективных альтернативных и экологически приемлемых ингибиторов гидратов является технологической проблемой для нефтегазовой отрасли. Ингибиторы добавляются в технологические линии для подавления образования гидратов. Впрыск химиката используется для трубопроводов протяженностью от 10 до 250 км.

Существуют две основные категории химических ингибиторов: термодинамические и низкодозированные ингибиторы гидратов.

Образование гидратов является серьезной проблемой для промышленности. Специалисты по обеспечению потока находились в поиске решений этой проблемы с момента разработки первых морских месторождений. Одно из первоначальных решений, которые до сих пор используется сегодня, это ввод химических веществ, называемых термодинамическими ингибиторами. Ингибиторы обычно представляют собой полярные растворители или ионные соли, которые не предотвращают образование гидратов, но вместо этого они снижают температуру пласта или увеличивают пластовое давление (т. е. изменяют фазовую оболочку, внутри которой гидрат стабилен).

Таким образом, они предотвращают попадание углеводородной жидкости в зону стабильности гидрата по всей длине трубопровода. Наиболее часто используемые термодинамические ингибиторы представляют собой метанол и этиленгликоль (МЭГ). Они до-

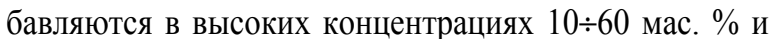
изменяют свойства жидкой и гидратной фаз, что позволяет сместить кривую диссоциации в область низких температур и высоких давлений.

Были проведены исследования образования гидратов (рис. 1). Справа от диаграммы гидраты не будут образовываться и система будет существовать в области флюидов (углеводородов и воды). Однако гидраты образуются в регионе слева от линии, обозначенной «Кривая образования гидратов», и следует принять меры по предотвращению их образования.

Эти условия показаны в виде почти горизонтальной линии, наложенной на условия образования гидратов, на рис. 1. На небольших расстояниях трубопровода (например, 11 км) от подводного устья скважины транспортируемый поток все еще сохраняет некоторое количество пластового тепла, так что гид- 
рат не образуется. Океан охлаждает протекающий поток, и примерно через 15 км единичная масса протекающего газа и попутной воды поступает в гидратную область слева от кривой образования гидрата, оставаясь в свободной области гидрата до 72 км. Такое расстояние может представлять несколько дней пребывания в водной фазе, так что гидраты, несомненно, будут образовываться, если не будут предприняты шаги по ингибированию.

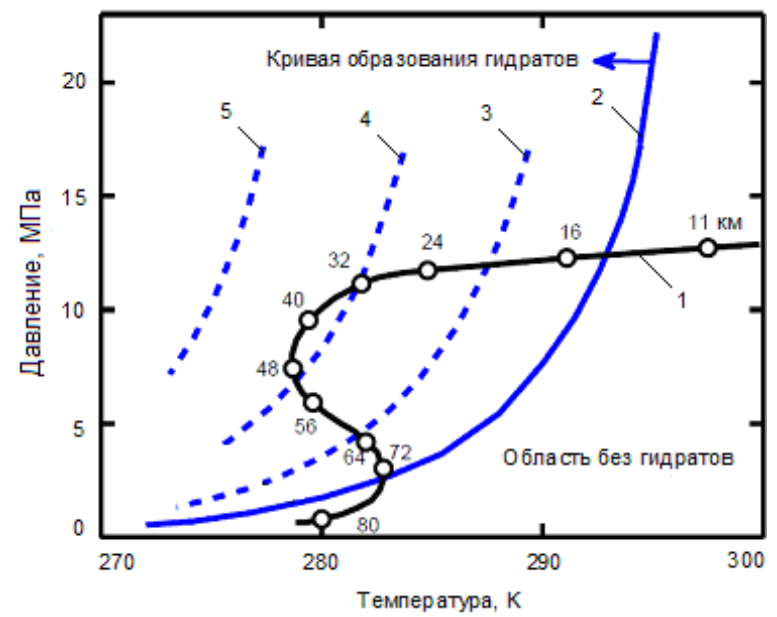

Рис. 1. Образование гидратов в трубопроводе: 1 - схема прокладки трубопровода; 2-5 - концентра иия метанола, соответственно, $\mathrm{Me}_{2}=0 \%$, $\mathrm{Me}_{3}=10 \%, \mathrm{Me}_{4}=20 \% \mathrm{u} \mathrm{Me}_{5}=30 \%$

Fig. 1. Formation of hydrates in the pipeline: 1 - pipeline scheme; 2-5-methanol concentration, respectively, $\mathrm{Me}_{2}=0 \%, \mathrm{Me}_{3}=10 \%, \mathrm{Me}_{4}=20 \%$ and $\mathrm{Me}_{5}=30 \%$

На рис. 1 на 40 км температура системы трубопровода находится в пределах нескольких градусов от температуры дна океана, так что примерно 23 мас. \% метанола требуется в фазе свободной воды для предотвращения образования гидратов и закупорки трубопроводов. Оборудование для закачки метанола недоступно в необходимых точках (от 15 до 72 км) вдоль трубопровода. Вместо этого метанол закачивается в трубопровод на подводном устье скважины. В случае трубопровода, показанного на рис. 1, достаточное количество метанола впрыскивается в устье скважины так, что в фазе свободной воды будет присутствовать избыток 23 мас. \% метанола по всей длине трубопровода. Концентрация метанола более 20 \% требуется для полного подавления образования гидратов в этой системе.

Когда испаренный метанол течет по трубопроводу, он растворяется в любой попутной воде или в воде, конденсированной из газа. Ингибирование гидрата происходит в свободной воде, обычно в скоплениях, где есть изменение в геометрии (например, изгиб или падение трубопровода вдоль впадины дна океана) или в некотором месте зародышеобразования (например, песок или сварной шов).

Ингибирование гидратов происходит в водной жидкости, а не в объеме паров. Хотя большая часть метанола растворяется в воде, значительное количество метанола либо остается в паре, либо растворяет- ся в жидкой углеводородной фазе. Даже если концентрация метанола в парах или жидких углеводородах мала, при небольших количествах воды большая часть метанола может потребляться паром или жидкими углеводородами, поскольку фракции углеводородной фазы намного больше, чем фракции водной фазы.

На рис. 1 показано, что температура газа увеличивается с 48 до 72 км с более теплыми (более мелкими) водными условиями. Однако на расстоянии от 72 до 80 км наблюдается вторая тенденция охлаждения изза эффекта расширения Джоуля-Томсона.

Термодинамические ингибиторы гидратов давно используются в промышленности и действуют как антифриз. Наиболее часто используемые - это метанол и моноэтиленгликоль.

Основной принцип использования термодинамических ингибиторов заключается в том, что он предотвращает образование гидратов путем смещения условий равновесия, при этом условия образования гидратов обеспечиваются при более низких температурах и повышенном давлении.

Таким образом, добавление метанола или моноэтиленгликоля смещает кривую равновесия влево, где достигается термодинамическое равновесие молекул жидкости и газа. В этом случае образование гидратов происходит при более низкой температуре и более высоком давлении, за счет этого снижается риск образования гидратов.

Они только изменяют условия температуры и давления, тем самым смещая рабочие условия из стабильной гидратной области, как показано на рис. 2.

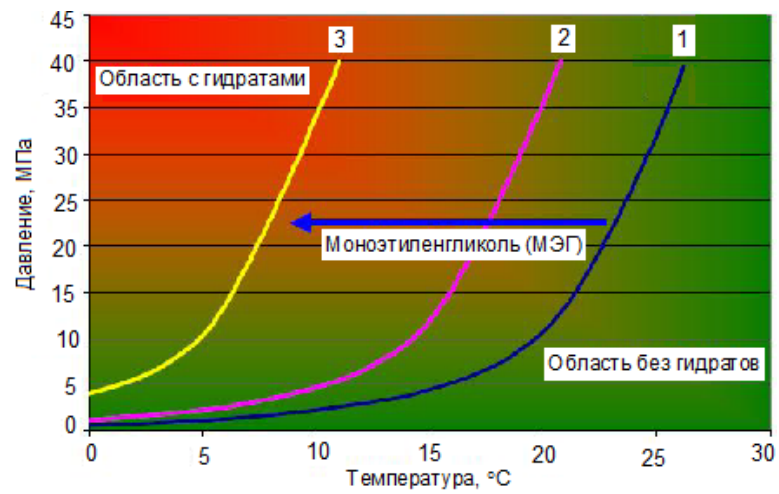

Pис. 2. Кривая равновесия гидрата газа: 1-3 - концентрация моноэтиленгликоля (МЭГ), соответственно, МЭ $\Gamma_{1}=0 \%, М Э \Gamma_{2}=20 \%$ и МЭ $\Gamma_{3}=40 \%$

Fig. 2. Equilibrium curve of gas hydrate: 1-3-the concentration of monoethylene glycol (MEG), respectively, $M E G_{1}=0 \%, M E G_{2}=20 \%$ and $M E G_{3}=40 \%$

Стратегия управления гидратами с использованием химикатов основана на непрерывном введении термодинамического ингибитора гидратов (как правило, моноэтиленгликоля) практически без изоляции подводной системы. Термодинамические ингибиторы редко используются в нефтяных системах, потому что для предотвращения гидратации потребуются высокие концентрации. Химикаты доставляются по трубопроводу малого диаметра в направлении, 
противоположном производственному потоку, из стационарных резервуаров на морском дне или на поверхности.

Ингибиторы обычно используются, когда обводненность в системе находится в диапазоне от 20 до 40 \% массы, но теоретически она может использоваться при всех обводнениях. Эффект переохлаждения метанола может достигать примерно $30{ }^{\circ} \mathrm{C}$ для 50 \% массы при давлениях от 0 до 17 МПа. Вязкость нефти всегда зависит от добавления ингибитора. При добавлении метанола полученная нефть будет более густой, а гликоля - более тонкой, но это сложно предсказать. Однако количество гидрата до $40 \%$ не обязательно дает измеримое изменение вязкости жидкости.

Гликоли широко используются в нефтегазовой промышленности, особенно при добыче природного газа. Когда моноэтиленгликоли используются в качестве ингибиторов газовых гидратов, их потеря в углеводородной фазе является наиболее существенным недостатком, особенно для метанола. Моноэтиленгликоли более предпочтительны, потому что их легче регенерировать: температура кипения моноэтиленгликоля выше, чем у метанола и потери на испарение незначительны.

Стратегия уменьшения гидратов основана на непрерывном введении термодинамического ингибито- ра гидратов (обычно моноэтиленгликоля) с небольшой изоляцией или без изоляции подводной системы. Сегодня моноэтиленгликоль представляет собой современный метод контроля гидратов. У них также, помимо предотвращения гидратов, МЭГ снижает скорость коррозии в трубопроводах из углеродистой стали, которые обычно используются, и хорошо подходит в качестве носителя ингибиторов коррозии и стабилизаторов рН. Моноэтиленгликоль также считается регенерируемым и экологически чистым из-за своих химических свойств и использования в замкнутой системе, где потери относительно невелики.

Кроме того, эффективное содержание ингибитора составляет менее $6 \%$ от подлежащего обработке газа. Системы регенерации обычно используются для кипячения воды в условиях, близких к атмосферному давлению. Рабочая температура зависит от необходимого содержания МЭГ. Метанол обычно не регенерируется.

Основные преимущества МЭГ включают в себя: надежное решение; замкнутый цикл; защита от коррозии; экологически чистый, нетоксичный, негорючий.

Краткое изложение преимуществ и ограничений при использовании химических ингибиторов для предотвращения гидратообразования представлено на рис. 3.

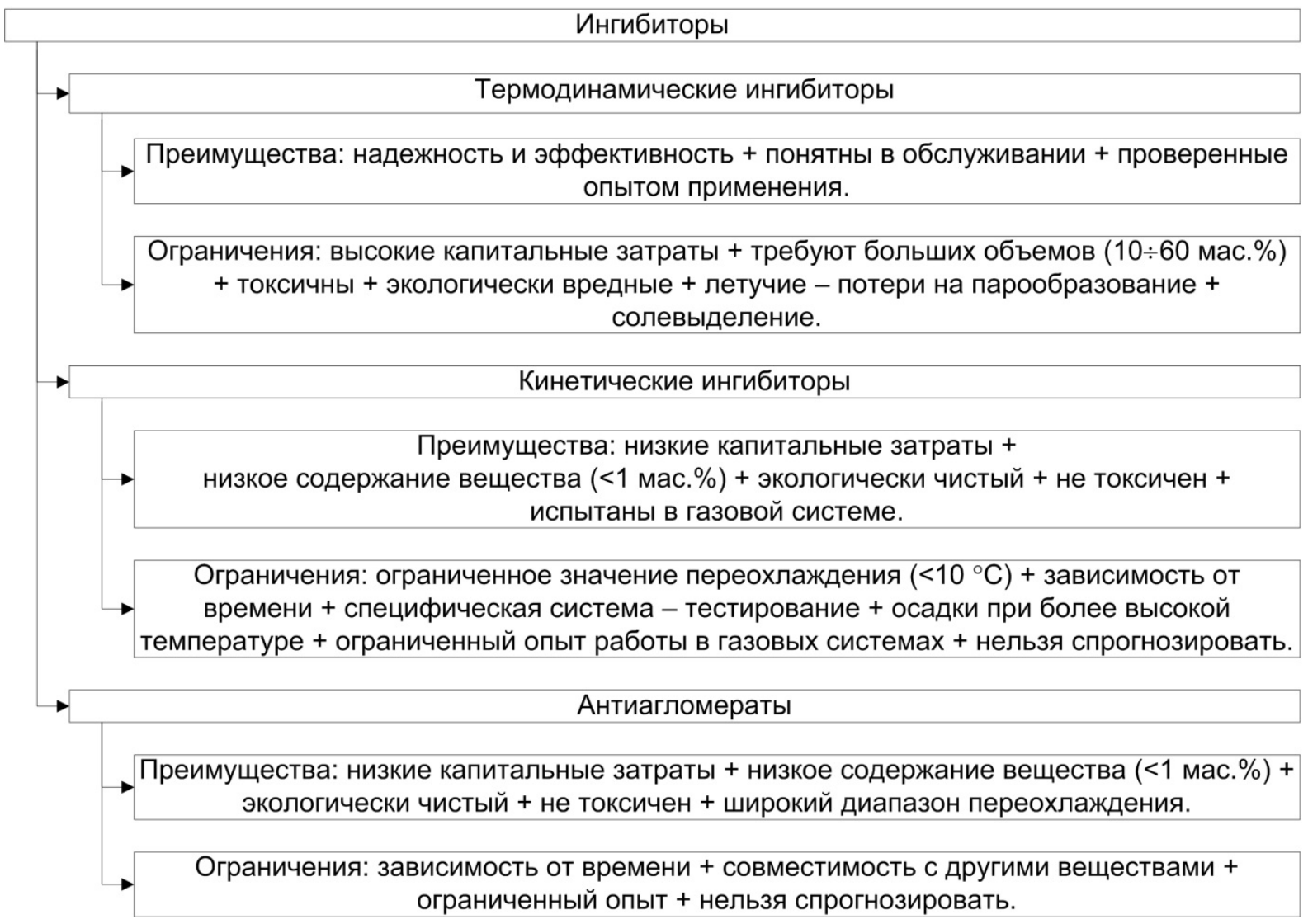

Pис. 3. Описание преимущчеств и ограничений химических ингибиторов

Fig. 3. Description of the benefits and limitations of chemical inhibitors 


\section{Программное обеспечение OLGA}

Существующее программное обеспечение [25-27] позволяет моделировать технологический процесс использования химических ингибиторов для предотвращения гидратообразования.

Программное обеспечение OLGA позволяет произвести анализ показателей перекачки нефти, природного газа и пластовой воды при многофазной транспортировке. Этот инструмент моделирования позволяет визуализировать ключевые аспекты динамических событий, отражающих реальность транспортировки углеводородов. Пакет OLGA позволил разрабатывать месторождения нефти и газа в более глубоких водах и дальше от берега, чем это было бы возможно без этой технологии.

Газовые гидраты вызывают серьезные и потенциально опасные проблемы обеспечения потока в производственных операциях. Типичная проблемная область - это многофазные транспортные линии от устья скважины к производственному объекту, где низкие температуры морского дна и высокое рабочее давление увеличивают риск засорения из-за образования гидрата газа.

Программное обеспечение OLGA позволяет прогнозировать риск образования гидратов и обеспечивает рабочий диапазон для предотвращения образования гидратов при транспортировке в скважину, на технологических объектах и при проектных вмешательствах в случае образования твердых образований.

Типичные случаи использования: предотвращение появления гидратов и оценка количества образовавшихся гидратов; использование гидратной суспензии; моделирование гидратной суспензии.

Газовый конденсат и вода при благоприятных условиях образуют гидратную пробку, которая закупоривает трубопровод. В морских трубопроводных системах при высоких давлениях и температурах очень часто создаются условия, при которых имеется высокая вероятность образования гидратов. Образовавшийся гидрат, представляющий собой по свойствам лед, невозможно удалить за короткое время, изза этого происходит снижение пропускной способности трубопровода или полная остановка транспортировки продукта.

Борьба с гидратообразованием - это закачка термодинамических ингибиторов, таких как метанол, моноэтиленгликоль, диэтиленгликоль и триэтиленгликоль. Ввиду того, что последние два представителя не обладают достаточной эффективностью, здесь будем рассматривать метанол $(\mathrm{Me})$ и моноэтиленгликоль (МЭГ).

Рассмотрим воздействие 20 \%-го раствора метанола на поток. На рис. 4 получили сдвиг кривой гидратообразования от первоначального положения, где не было никакого воздействия на систему.

Теперь уменьшим содержание метанола в растворе до $10 \%$ и посмотрим, как будет вести себя кривая образования гидратов. Даже в случае с 10\%-м раствором метанола видим, что гидраты еще не образуются. Теперь смоделируем ситуацию с 5\%-м раствором (рис. 4) и убедимся в обратном.
Проводя подобные операции с разными концентрациями метанола, в итоге можем получить вполне ожидаемый результат: чем больше концентрация, тем больше линия смещается влево (рис. 4).

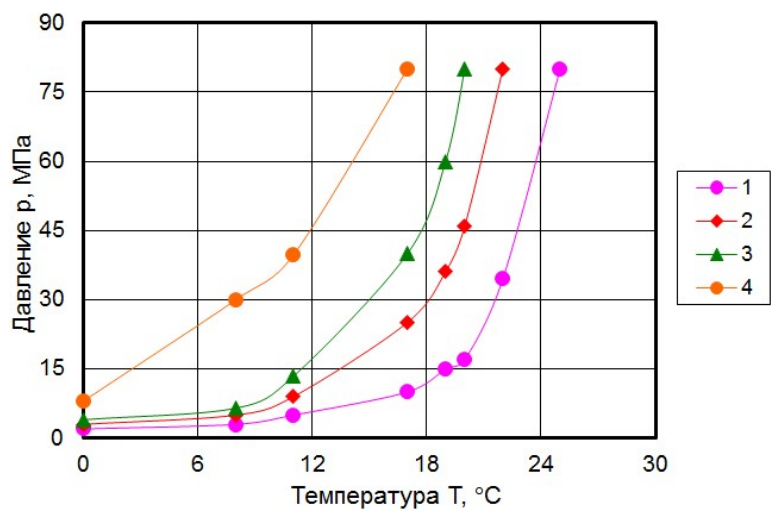

Pис. 4. Кривые гидратообразования при различных концентрациях метанола (Ме): 1 - без ингибиторов, концентрация метанола Ме ${ }_{1}=0 \%$; 2 - кониентрация метанола $\mathrm{Me}_{2}=10 \% ; 3$ - концентрация метанола $\mathrm{Me}_{3}=20 \%$; 4 - концентрация метано$\mathrm{ra} \mathrm{Me}_{4}=25 \%$

Fig. 4. Hydrate formation curves at various concentrations of methanol (Me): 1 - without inhibitors, methanol concentration $\mathrm{Me}_{1}=0 \%$; 2 - methanol concentration $\mathrm{Me}_{2}=10 \% ; 3$ - methanol concentration $\mathrm{Me}_{3}=20 \% ; 4-$ methanol concentration $\mathrm{Me}_{4}=25 \%$

Аналогичные кривые гидратообразования можно получить в программе OLGA при различных концентрациях моноэтиленгликоля (рис. 5).

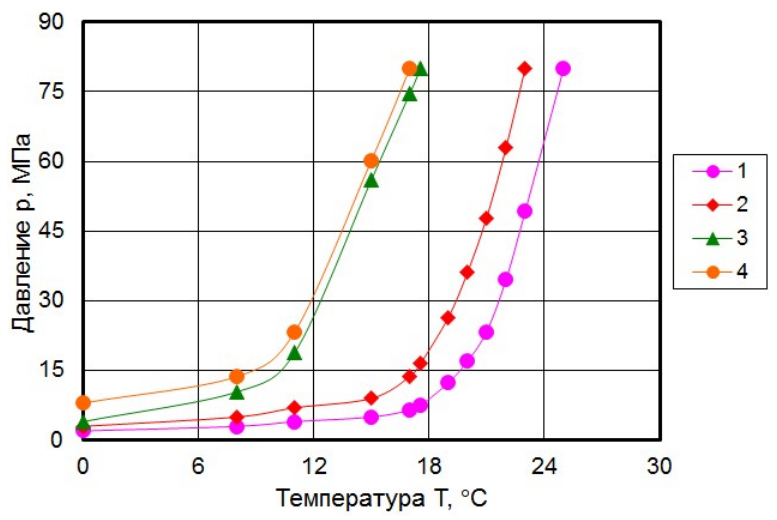

Рис. 5. Кривые гидратообразования при различных концентрациях моноэтиленгликоля (МЭГ): 1 - без ингибиторов, кониентрация моноэтиленгликоль МЭ $\Gamma_{1}=0 \% ; 2$ - концентрация МЭ $\Gamma_{2}=10 \% ; 3-$ концентраиия $М Э \Gamma_{3}=20 \%$; 4 - кониентрация $M Э \Gamma_{4}=25 \%$

Fig. 5. Hydrate formation curves at various concentrations of monoethylene glycol (MEG): 1 - without inhibitors, the concentration of monoethylene glycol $M E G_{1}=0 \% ; 2$ - concentration of $M E G_{2}=10 \% ; 3$ concentration of $M E G_{3}=20 \% ; 4$ - concentration of $M E G_{4}=25 \%$

Рассмотренный метод предотвращения газовых гидратов - закачка термодинамических ингибиторов, применялся для разработки месторождения в Арктике. Бы- 
ло установлено, что оптимальное решение для борьбы с гидратами - это закачка термодинамических ингибиторов, таких как метанол или моноэтиленгликоль.

\section{Заключение}

Разработка запасов арктического шельфа является одной из перспективных областей, через которые можно вести добычу нефти и газа. Арктический шельф может стать важным источником долгосрочного роста добычи. Однако сложные ледовые и погодные условия и отсутствие инфраструктуры требуют другого подхода и технологий.

Добыча газа в Арктике связана с необходимостью транспортировки углеводорода на большие расстояния, в результате чего транспортируемый природный газ и вода образуют газовые гидраты. Образование гидрата с последующим накоплением создает большие агломерации. В результате это приводит к огра-

\section{СПИСОК ЛИТЕРАТУРЫ}

1. Егоров А.В., Римский-Корсаков Н.А., Лискин В.А. Методы и средства обнаружения и исследования газовых гидратов // Coвременные методы и средства океанологических исследований (МСОИ-2019). - М.: Институт океанологии им. П.П. Ширшова РАН, 2019. - С. 192-195.

2. Рябов А.Д. Современный подход к образованию газовых гидратов: прогнозирование, методы предупреждения и борьбы // Проблемы геологии и освоения недр. - Томск: Национальный исследовательский Томский политехнический университет, 2019. - C. $166-168$.

3. Широбоков О.С., Свалова М.В. Газовые гидраты в газопроводах природного газа // Актуальные проблемы современной когнитивной науки. - Уфа: Аэтерна, 2019. - С. 15-20.

4. Бухгалтер Э.Б. Метанол и его использование в газовой промышленности. - М: Недра, 1986. - 283 с.

5. Истомин В.А. Предупреждение и ликвидация газовых гидратов в системах сбора и промысловой обработки газа и нефти. - М.: ВНИИГАЗ, 1990. - 213 с.

6. Бухгалтер Э.Б., Ильякова Е.Е. Нормативные аспекты экологического мониторинга при морской нефтегазодобыче в Арктике // Вести газовой науки. - 2013. - № 2 (13). - С. 82-87.

7. Волков П.В. Выбор способа при теплофизическом воздействии для предотвращения гидратообразования при подготовке газа и конденсата // Проблемы геологии и освоения недр. Томск: Национальный исследовательский Томский политехнический университет, 2019. - Т. 1. - С. 74-75.

8. Перспективы применения технологий хранения и транспортировки углеводородного сырья на основе газовых гидратов в условиях Арктики / М.Е. Семенов, А.С. Портнягин, В.В. Корякина, И.К. Иванова // Теплофизика и энергетика арктических и субарктических территорий. - М.: Национальный исследовательский университет МЭИ, 2019. - С. 246-251.

9. Gudmundsson J. Hydrate non-pipeline technology // Proceedings of the 4th International Conferences on Gas Hydrates. Yakohama, Japan, May 19-23, 2002. - P. 997-1002.

10. Макиян Т.Г., Землянов Е.Г. Расчет количества ингибиторов в модели газопровода // Наука и образование в Арктическом регионе. - Мурманск: Мурманский государственный технический университет, 2018. - С. 73-75.

11. Брилл Дж.П., Мукерджи Х. Многофазный поток в скважинах. Ижевск: Институт компьютерных исследований, 2006. - 384 с.

12. Фаресов А.В., Шагапов А.В., Пономарев А.И. Исследование эффективности ингибиторов гидратообразования кинетического типа // Нефтегазовое дело. - 2013. - Т. 11. - № 4. - С. 86-95.

13. Синергетические эффекты при ингибировании образования газовых гидратов в многокомпонентных системах / А.П. Семенов, Р.И.М. Мендгазиев, А.С. Стопорев, П.А. Гушин, В.С. Якушев, В.А. Винокуров // От синтеза полиэтилена до стереодивергентности: развитие химии за 100 лет. - Пермь: Перм- ничению и блокировке трубопровода. Ясно, что они будут препятствовать потоку углеводородов. Чтобы избежать этого, все больше и больше внимания уделяется разработке методов обеспечения потока, чтобы предотвратить образование гидратных пробок.

Математическое моделирование (использование программного комплекса OLGA) позволяет прогнозировать риск образования гидратов и обеспечивает рабочий диапазон для предотвращения образования гидратов при транспортировке в скважину, на технологических объектах и при проектных вмешательствах в случае образования твердых образований.

Установлена эффективность добавления 10 \%-го раствора метанола для предотвращения гидратообразования как наиболее выгодное решение для Арктики по сравнению с другими технологиями и другими концентрациями соответственно.

ский государственный национальный исследовательский университет, 2018. - С. 252-254.

14. Application of various water soluble polymers in gas hydrate inhibition / M.S. Kamal, I.A. Hussein, A.S. Sultan, N. Solms // Renewable and Sustainable Energy Reviews. - 2016. - № 60. - P. 206-225.

15. Nakai S. Development of natural gas hydrate (NGH) supply chain // Proceedings of the 25th world gas conferences. - Kuala Lumpur, Malaysia, June 4-8, 2012. - P. 367-375.

16. Maruyama T. Effects of guest gas on pelletizing performance of natural gas hydrate $(\mathrm{NGH})$ pellets // Proceedings of the 7th ICGH 2011. - Edinburgh, Scotland, United Kingdom, July 17-21, 2011. - P. 181-187.

17. Horiguchi $\mathrm{K}$. Completion of natural gas hydrate (NGH) overland transportation demo project // Proceedings of the 7th International Conference on Gas Hydrates (ICGH 2011). - Edinburgh, Scotland, United Kingdom, July 17-21, 2011. - P. 401.

18. Хилик Е.Д. Технология холодной перекачки Cold Flow // Moлодой ученый. - 2019. - № 4 (242). - С. 74-75.

19. Raoelison R.N., Verdy C., Liao H. Cold gas dynamic spray additive manufacturing today: deposit possibilities, technological solutions and viable applications // Materials and Design. 2017. - V. 133. - P. 266-287.

20. Decomposition kinetics and self-preservation of methane hydrate particles in crude oil dispersions: experiments and theory / A.A. Sizikov, V.A. Vlasov, A.S. Stoporev, A.Yu. Manakov // Energy and fuels, American Chemical Society. - 2019. - V. 33. № 12. - P. 12353-12365.

21. Lee J., Jin Y.K., Seo Y. Characterization of cyclopentane clathrates with gaseous guests for gas storage and separation // Chemical engineering journal. - 2018. - V. 338 . - P. 572-578.

22. Thermodynamic and kinetic analysis of gas hydrates for desalination of saturated salinity water / S.D. Seo, S.Y. Hong, A.K Sum, K.H. Lee, J.D. Lee, B.R. Lee // Chemical engineering journal. - 2019. - V. 370. - P. 980-987.

23. Hammerschmidt E.G. Formation of gas hydrates in natural gas transmission lines // Industrial and Engineering Chemistry. 1934. - V. 26. - № 8. - P. 851-855.

24. Carroll J.J. Natural gas hydrates. A guide for engineers. - Calgary, Canada: Elsevier Science and Technology Books, 2009. - 276 p.

25. Сравнительный анализ результатов экспериментальных исследований вертикальных газожидкостных потоков и расчетов по программе OLGA / Д.В. Изюмченко, И.В. Стоноженко, К.Н. Гужов, В.А. Сулейманов, О.В. Бузинова, О.В. Николаев // Вести газовой науки. - 2016. - № 2 (26). - С. 91-95.

26. OLGA 6 GUI: user manual. - Kjeller, Norway: SPT group AS, 2008. $-240 \mathrm{p}$

27. Cowie S., Zolotukhin A.B. Hydrate plugs in subsea pipelines and non-invasive methodology for localization. - Stavanger, Norway: University of Stavanger, 2013. - $78 \mathrm{p}$

Поступила: 27.07.2020 2. 


\section{Информация об авторах}

Китаев С.B., доктор технических наук, профессор, профессор кафедры транспорта и хранения нефти и газа Уфимского государственного нефтяного технического университета.

Колотилов Ю.В., доктор технических наук, профессор, профессор кафедры высшей и прикладной математики Астраханского государственного технического университета.

Плотников А.Ю., аспирант кафедры высшей и прикладной математики Астраханского государственного технического университета.

Ковалев А.А., аспирант кафедры высшей и прикладной математики Астраханского государственного технического университета.

Шейхгасанов Ш.К., аспирант кафедры высшей и прикладной математики Астраханского государственного технического университета. 
UDC 621.644 .029

\title{
STUDY OF EFFICIENCY OF HYDRATE FORMATION INHIBITORS IN THE PROCESS OF PRODUCTION AND TRANSPORT OF HYDROCARBONS IN MARINE CONDITIONS
}

\author{
Sergey V. Kitaev 1 , \\ svkitaev@mail.ru
}

Yury V. Kolotilov', kolotilov_yury@mail.ru

Anton Yu. Plotnikov2, plotnikovyurii@rambler.ru

\author{
Adel A. Kovalev², \\ truenerd123@gmail.com \\ Shamsutdin K. Sheikhgasanov², \\ sheih0113@mail.ru \\ 1 Ufa State Petroleum Technological University, \\ 1, Kosmonvtov street, Ufa, 450062, Russia. \\ 2 Astrakhan State Technical University, \\ 16, Tatishchev street, Ufa, 414056, Russia.
}

The relevance of the research is caused by the need to develop effective methods to prevent hydrate formation in underwater gas pipelines. The rapid formation of gas hydrates is promoted by high pressure and low temperature in deep-sea underwater structures, which is one of the most difficult problems in ensuring the operability of the pipeline. The main direction of ensuring trouble-free operation of pipelines is to support the processes of using hydrate inhibitors during the operation of hydrocarbon transport facilities, taking into account their advantages and disadvantages.

The main aim of the research is to develop a method for evaluating the effectiveness of hydrate inhibitors in production and transport of hydrocarbons in marine conditions.

The objects of the research are performance indicators for implementation of hydrate inhibitor use methods with an assessment of the risk associated with hydrate formation, which helps prevent hydrate blockage and ensure equipment integrity.

Methods. To increase the reliability of the interpretation of methods for the use of hydrate inhibitors, it is proposed to use the OLGA software, which is a tool for modeling the analysis of pumping oil, natural gas, and produced water during multiphase transportation. This modeling tool allows you to visualize key aspects of dynamic events that reflect the reality of hydrocarbon transportation. The OLGA software suite has allowed developing oil and gas fields in deeper waters and further offshore than it was possible without this technology.

Results. Using OLGA software, it was possible to consider existing methods for preventing hydrate formation and concluded that cold flow technology, as well as the addition of a $10 \%$ methanol solution, are the most beneficial solution for the Arctic compared to other technologies and other concentrations, respectively.

\section{Key words:}

Offshore pipeline, inhibitor, hydrate formation, software, concentration, pressure, temperature.

\section{REFERENCES}

1. Egorov A.V., Rimsky-Korsakov N.A., Liskin V.A. Metody i sredstva obnaruzheniya i issledovaniya gazovykh gidratov [Methods and means of detecting and studying gas hydrates]. Sovremennye metody i sredstva okeanologicheskikh issledovaniy (MSOI2019) [Modern methods and means of oceanological research (MSOI-2019)]. Moscow, Institute of Oceanology im P.P. Shirshova RAN Publ., 2019. pp. 192-195.

2. Ryabov A.D. Sovremenny podkhod k obrazovaniyu gazovykh gidratov: prognozirovanie, metody preduprezhdeniya i borby [A modern approach to the formation of gas hydrates: forecasting, methods of prevention and control]. Problemy geologii i osvoyeniya nedr [Problems of geology and subsoil development]. Tomsk, National Research Tomsk Polytechnic University Publ., 2019. pp. 166-168.

3. Shirobokov O.S., Svalova M.V. Gazovye gidraty v gazoprovodakh prirodnogo gaza [Gas hydrates in natural gas pipelines]. Aktualnye problemy sovremennoy kognitivnoy nauki [Actual problems of modern cognitive science]. Ufa, Aeterna Publ., 2019. pp. 15-20.

4. Bukhgalter E.B. Metanol i ego ispolzovanie $v$ gazovoy promyshlennost $i$ [Methanol and its use in the gas industry]. Moscow, Nedra Publ., 1986. 283 p.
5. Istomin V.A. Preduprezhdenie i likvidatsiya gazovykh gidratov $v$ sistemakh sbora i promyslovoy obrabotki gaza i nefti [Prevention and elimination of gas hydrates in the systems of collection and field processing of gas and oil]. Moscow, VNIIGAZ Publ., 1990. $213 \mathrm{p}$.

6. Bukhgalter E.B., Ilyakova E.E. Regulatory aspects of environmental monitoring during offshore oil and gas production in the Arctic. Vesti gazovoy nauki, 2013, no. 2 (13), pp. 82-87. In Rus.

7. Volkov P.V. Vybor sposoba pri teplofizicheskom vozdeystvii dlya predotvrashcheniya gidratoobrazovaniya pri podgotovke gaza i kondensata [The choice of the method under thermophysical exposure to prevent hydrate formation during the preparation of gas and condensate]. Problemy geologii $i$ osvoyeniya nedr [Problems of geology and subsoil development]. Tomsk, National Research Tomsk Polytechnic University Publ., 2019. Vol. 1, pp. 74-75.

8. Semenov M.E., Portnyagin A.S., Koryakina V.V., Ivanova I.K. Perspektivy primeneniya tekhnologiy khraneniya i transportirovki uglevodorodnogo syrya na osnove gazovykh gidratov v usloviyakh Arktiki [Prospects for application of technologies for storage and transportation of hydrocarbons based on gas hydrates in the Arctic]. Teplofizika i energetika arkticheskikh i subarkticheskikh territoriy [Thermophysics and energy of the Arctic and subarctic terri- 
tories]. Moscow, National Research University MEI Publ., 2019. pp. 246-251.

9. Gudmundsson J. Hydrate non-pipeline technology. Proceedings of the 4th International Conferences on Gas Hydrates. Yakohama, Japan, May 19-23, 2002. pp. 997-1002.

10. Makiyan T.G., Zemlyanov E.G. Raschet kolichestva ingibitorov v modeli gazoprovoda [Calculation of the number of inhibitors in a gas pipeline model]. Nauka i obrazovanie $v$ Arkticheskom regione [Science and Education in the Arctic Region]. Murmansk, Murmansk State Technical University Publ., 2018. pp. 73-75.

11. Brill Dzh.P., Mukerdzhi Kh. Mnogofazny potok $v$ skvazhinakh [Multiphase flow in wells]. Izhevsk, Institute for Computer Research Publ., 2006. 384 p.

12. Faresov A.V., Shagapov A.V., Ponomarev A.I. Investigation of the effectiveness of kinetic-type hydrate inhibitors. Oil and Gas Business, 2013, vol. 11, no. 4, pp. 86-95. In Rus.

13. Semenov A.P., Mendgaziev R.I.M., Stoporev A.S., Gushchin P.A., Yakushev V.S., Vinokurov V.A. Sinergeticheskie effekty pri ingibirovanii obrazovaniya gazovykh gidratov $\mathrm{v}$ mnogokomponentnykh sistemakh [Synergistic effects in inhibiting the formation of gas hydrates in multicomponent systems]. Ot sinteza polietilena do stereodivergentnosti: razvitie khimii za 100 let [From the synthesis of polyethylene to stereo-divergence: the development of chemistry over 100 years]. Perm, Perm State National Research University Publ., 2018. pp. 252-254.

14. Kamal M.S., Hussein I.A., Sultan A.S., Solms N. Application of various water soluble polymers in gas hydrate inhibition. Renewable and Sustainable Energy Reviews, 2016, no. 60, pp. 206-225.

15. Nakai S. Development of natural gas hydrate (NGH) supply chain. Proceedings of the 25th world gas conferences. Kuala Lumpur, Malaysia, June 4-8, 2012, pp. 367-375.

16. Maruyama T. Effects of guest gas on pelletizing performance of natural gas hydrate $(\mathrm{NGH})$ pellets. Proceedings of the 7th $\mathrm{ICGH}$ 2011. Edinburgh, Scotland, United Kingdom, July 17-21, 2011, pp. 181-187.

17. Horiguchi K. Completion of natural gas hydrate (NGH) overland transportation demo project. Proceedings of the 7th International
Conference on Gas Hydrates (ICGH 2011). Edinburgh, Scotland, United Kingdom, July 17-21, 2011, p. 401.

18. Khilik E.D. Cold Flow Technology. Young Scientist, 2019, no. 4 (242), pp. 74-75. In Rus.

19. Raoelison R.N., Verdy C., Liao H. Cold gas dynamic spray additive manufacturing today: Deposit possibilities, technological solutions and viable applications. Materials and Design, 2017, vol. 133, pp. 266-287.

20. Sizikov A.A., Vlasov V.A., Stoporev A.S., Manakov A.Yu. Decomposition kinetics and self-preservation of methane hydrate particles in crude oil dispersions: experiments and theory. Energy and fuels, American Chemical Society, 2019, vol. 33, no. 12, pp. $12353-12365$.

21. Lee J., Jin Y.K., Seo Y. Characterization of cyclopentane clathrates with gaseous guests for gas storage and separation. Chemical engineering journal, 2018, vol. 338, pp. 572-578.

22. Seo S.D., Hong S.Y., Sum A.K., Lee K.H., Lee J.D., Lee B.R. Thermodynamic and kinetic analysis of gas hydrates for desalination of saturated salinity water. Chemical engineering journal, 2019, vol. 370, pp. 980-987.

23. Hammerschmidt E.G. Formation of gas hydrates in natural gas transmission lines. Industrial and Engineering Chemistry, 1934, vol. 26 , no. 8 , pp. 851-855.

24. Carroll J.J. Natural gas hydrates. A guide for engineers. Calgary, Canada, Elsevier Science and Technology Books Publ., 2009. $276 \mathrm{p}$.

25. Izyumchenko D.V., Stonozhenko I.V., Guzhov K.N., Suleimanov V.A., Buzinova O.V., Nikolaev O.V. Comparative analysis of the results of experimental studies of vertical gas-liquid flows and calculations using the OLGA program. Vesti gazovoy nauki, 2016, no. 2 (26), pp. 91-95. In Rus.

26. OLGA 6 GUI: user manual. Kjeller, Norway, SPT group AS Publ., 2008. $240 \mathrm{p}$.

27. Cowie S., Zolotukhin A.B. Hydrate plugs in subsea pipelines and non-invasive methodology for localization. Stavanger (Norway), University of Stavanger Publ., 2013. 78 p.

Received: 27 July 2020.

\section{Information about the authors}

Sergei V. Kitaev, Dr. Sc., professor, Ufa State Petroleum Technological University.

Yury V. Kolotilov, Dr. Sc., professor, Astrakhan State Technical University.

Anton Yu. Plotnikov, graduate student, Astrakhan State Technical University.

Adel A. Kovalev, graduate student, Astrakhan State Technical University.

Shamsutdin K. Sheikhgasanov, graduate student, Astrakhan State Technical University. 